\title{
A Fast Predicting Neural Fuzzy Model for Suspended Solid Removal Efficiency in Multimedia Filter
}

\author{
Rusul Naseer', Alaa' Abdulrazaq Jassim ${ }^{1}$, Saad AbuAlhail ${ }^{2}$ \\ ${ }^{1}$ Department of Chemical Engineering, Faculty of Engineering, Basrah University, Basrah, Iraq; ${ }^{2}$ Department of Civil Engineering, \\ Faculty of Engineering, Basrah University, Basrah, Iraq. \\ Email: RUSALNASEER@yahoo.com
}

Received August $4^{\text {th }}, 2010$; revised September $6^{\text {th }}, 2010$; accepted September $10^{\text {th }}, 2010$.

\begin{abstract}
Modeling of filter performance is very difficult because of complexity of the defining physical and chemical events in the filtration system whereas the knowledge of functionality of filter coefficient. The main objective of this study is to predict the performance of multimedia filter and to evaluate both the initial and transient stage of suspended solid removal efficiency depending on experimental data. Fuzzy logic has been used to build a model of multi input and one output (MISO) for the removal efficiency of multimedia filter which it consists from sand and granular activated carbon (GAC) mediums. The control parameters of (FLC) of Sugeno model are seven parameters which are media depths, media grains size for both sand and GAC, filtration rate, diameter of suspension particle, feed concentration, and operation time. The output parameter is removal efficiency of media filter whereas these data are collocated from pilot scale deep bed filter, thus, the removal efficiency of filter was modeled by 575 rules as a function of different control parameters. An adaptive of neuron fuzzy inference system (ANFIS) had used to simulate the experimental data. The simulation results were evaluated using mean absolute percentage error (MAPE), whereas the results showed that the prediction of ANFIS model has a good agreement with experimental data when the MAPE is equal to 7.0458 and fuzzy rule -based modeling proved a reliable and flexible tool to study the performance of multimedia filter. The conclusion showed that there is a relationship between flow rate, effective size and optimum bed depth for all filter media, the increment of effective size generates a higher value of optimum filter bed depth for a lower value of filtration rate. It was concluded that the optimal removal efficiency (95-100) achieved by (0.5-0.7 mm) grain size of sand, (1.5-1.9) $\mathrm{mm}$ grain size of granular activated carbon (GAC), with media depths should range from 0.3 to $0.6 \mathrm{~m}$.
\end{abstract}

Keywords: Multimedia Filter, Sand Filtration, Removal Efficiency, Fuzzy Logic, Suspended Solid

\section{Introduction}

Deep bed filtration is an effective process in removing particles from range $0.01(\mu \mathrm{m})$ to $100(\mu \mathrm{m})$ in size [1] Removal of these particles by deep bed filtration involves complex mechanisms .First; particles in suspension are transported near filter grains by mechanisms such as sedimentation, interception, diffusion, inertia and hydrodynamic effect. The effective removal of these particles depends on the attachment mechanism, which depends on the surface forces acting between particles and filter grains. The factors, which affect these forces eventually, it will affect of the performance of deep bed filtration. Generally, several mathematical filtration models have been proposed to describe Dispersed particles removal by filter whereas macroscopic and microscopic theories are widely ac- cepted. In the macroscopic approach, first order kinetics in the removal of particulate is assumed, whereas the microscopic approach takes into account single collector efficiencies. Filtration equations describing the deep bed filtration was proposed by [2]. Similarly, there are many other models available in the literature including in [3-5]. These models require data for characteristics of filter media, type of flow and liquid. In last decade, fuzzy logic and neural network has been employed to model in some complex environmental processes such as water and wastewater treatment. Fuzzy logic provides a language with a syntax and semantics to translate qualitative knowledge into a Numerical reasoning. The term computing with words has been introduced by [6] to explain the notion of reasoning linguistically rather than with numerical quantities. Such reasoning has central importance for many emer- 
ging technologies related to engineering and the sciences. Fuzzy and neural models have shown great advantages in the simulation, prediction and controlling of the medical diagnosis, information technology, water quality, reliability analysis and many other industrial applications, [7] Fuzzy modeling was used to predict estuary quality in [8], prediction of evaporation [9], prediction of Consumption Istanbul city using fuzzy logic approach [10], prediction of water level in reservoir [11], modeling of anaerobic digestion of primary sedimentation sludge [12]. In this study, the performance of multimedia filter (sand and GAC) filters was investigated and predicated as a function of media characteristics, operation condition and the quality of row water by a neural fuzzy model based on adaptive neuron-fuzzy inference system (ANFIS) model which is proposed in this work.

\section{Material and Methods}

\subsection{Experimental Set up}

Filtration Process was carried out by a pilot laboratory filter which is used to collect the data in this work. The laboratory scale Deep bed filter column has been designed to operate identically to full scale granular filters. Using deferent filter media with different bed depth tests on this unit to provide operational data which was scaled up to full size. The media in each filter was supported on a PVC orifice plate drilled with $5 \mathrm{~mm}$ holes and covered by a wire mesh to prevent sand passing through the orifices. A scale was made in front of tube column to estimate bed height. The laboratory filter was used to measure the experimental removal efficiency of suspension clay in multimedia filter and concentration profiles through the filter bed. The Deep Bed Filter Column is a clear acrylic unit mounted in a floor standing framework approximately $2 \mathrm{~m}$ high with flanged end pieces to allow easy access. The medium is supported on a corrosion resistant gauze mesh below is packed $1 \mathrm{~kg}$ of $0.01 \mathrm{~m}$ Ballotini to ensure good wash water distribution . Slotted sampling tubes inserted through the wall penetrate into the media, and are fitted with control valves so that suspension samples can be taken are kinetically. Plain tubes also penetrate the medium through the wall to transmit pressure to a manometer system. These sampling and manometer probes are located at $0.02 \mathrm{~m}$ depth intervals, but staggered in position, over $0.8 \mathrm{~m}$ depth which is 0.2 $\mathrm{m}$ GAC media over $0.6 \mathrm{~m}$ sand media and. Consequently as shown in Figure 1 where Deep bed filter consist of pump, sump tank, flow controller, rotameter, control valves, tubing, sampling tubes and bank of water differential manometers as shown in figure below.

\subsection{Model Architecture and Model Components}

The schematic architecture of the neural fuzzy model is depicted in Figure 2. It consists of the five key components: inputs and outputs database and preprocessor, a fuzzy system generator, a fuzzy inference system, and, an adaptive neural network representing the fuzzy system. The input and output parameters are selected or generated from the major parameters that is influence on removal efficiency which are flow rate, influent concentration of suspended solid, filter depth, types of media, grain size, diameter of suspension particle and operating time. One output parameter is the removal efficiency of filter. Table 1 showed the range of each input that is used in this work.

\section{Adaptive Network-Based Fuzzy Inference System (ANFIS)}

In this work, both the (FLC) and ANNS have been employed together to design a neuron -Fuzzy logic controller. A fuzzy system with learning ability has been constructed and is trained directly from the input-output data of the plant. Since the ANFIS has the property of learning; membership functions and fuzzy

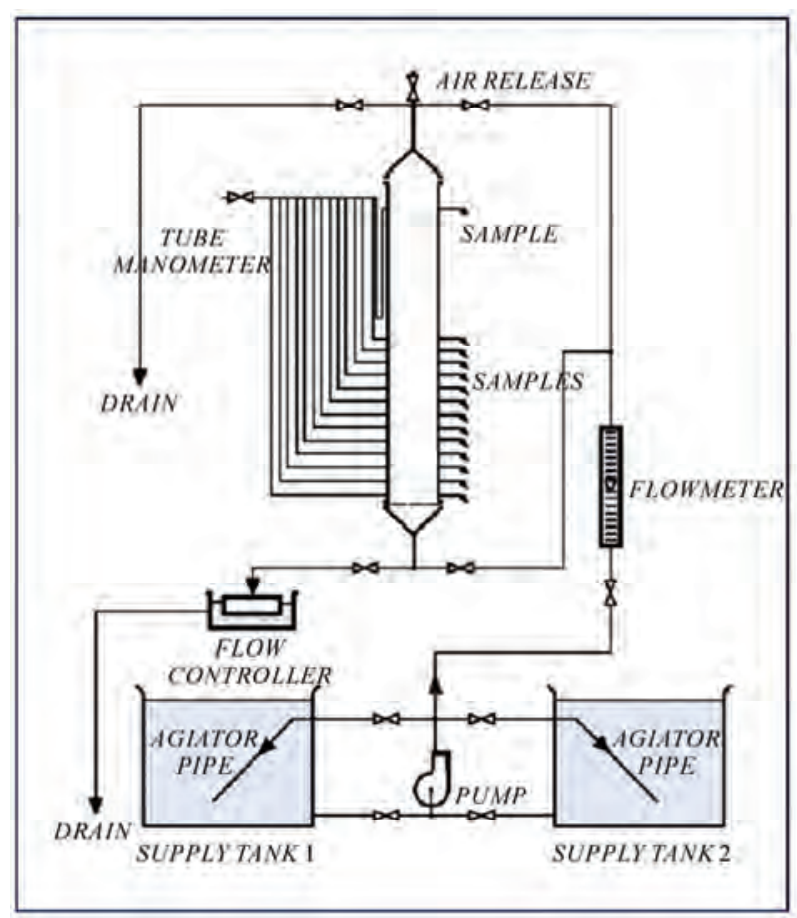

Figure 1. Laboratory pilot filtration unit schematic. 


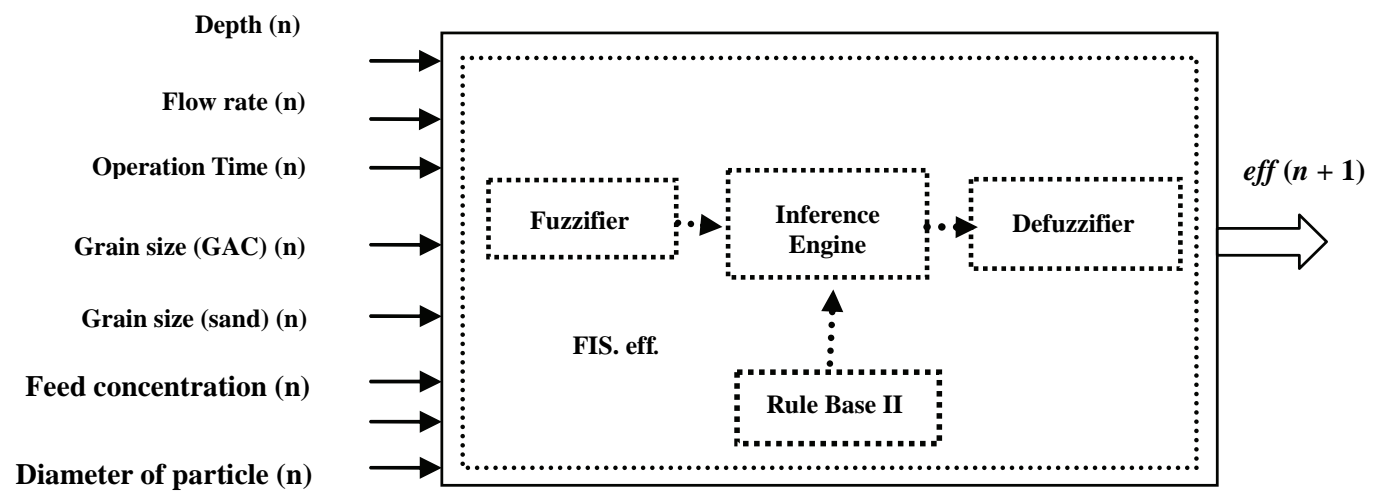

Figure 2. Corresponding ANFIS schematic architecture.

Table 1. Ranges of input variables.

\begin{tabular}{cc}
\hline Input variable & The range \\
\hline Flow rate $\left(\mathrm{cm}^{3} / \mathrm{min}\right)$ & $500-1300$ \\
Feed concentration $(\mathrm{mg} / \mathrm{l})$ & $2750-34000$ \\
Sand grain size $(\mathrm{mm})$ & $0.5-1.18$ \\
GAC grain size $(\mathrm{mm})$ & $1.5-2.36$ \\
Sand depth $(\mathrm{m})$ & $0.1-0.7$ \\
GAC depth $(\mathrm{m})$ & 0.2 \\
Diameter of particle $(\mu \mathrm{m})$ & $0.01-100$ \\
Operation time $(\mathrm{sec})$ & $0-8400$ \\
\hline
\end{tabular}

rules of the controller can be tuned automatically by the learning algorithm. Learning is based on the performance error, which is evaluated by comparing the process output with the desired or required output. ANFIS provides two main perspectives: one is to transform human knowledge or experience into the rule base and database of a fuzzy inference system and second is to tune the membership functions resulting to minimize the output error measure or maximize performance index. ANFIS can also act as for building a set of fuzzy if-then rules with appropriate membership functions to generate the stipulated input-output pairs [13].

\subsection{Fuzzy Rule-Based Modeling}

Fuzzy sets are the sets with boundaries that are not precise. A fuzzy set is an extension of the traditional set theory (in which $\mathrm{x}$ is either a member of set $\mathrm{A}$ or not) in that an $x$ can be a member of set A with a certain degree of membership $\mu$ among the various types of fuzzy sets. A fuzzy number describes the relationship between an uncertain quantity $\mathrm{x}$ and a membership function $\mu$ which ranges between 0 and 1 it is assumed that the fuzzy inference system under consideration has two inputs and one output. The rule basis contains two fuzzy if- then rules of Takagi and Surgeon's type as follows [14]:

If $x$ is $A$ and $y$ is $B$ then $Z$ is $f(x . y)$

Where, $A$ and $B$ are the fuzzy sets in the antecedents and $z=f(x . y)$ is a crisp function in the consequent. $f(x . y)$ is usually a polynomial for the input variables $x$ and $y$. But it can also be any other function for approximate description of the output of the system within the fuzzy region as specified by the antecedent. When $f(x . y)$ is a constant, a zero order, Sugeno fuzzy model is formed, which may be considered to be a special case of Mamdani fuzzy inference system where each rule consequence is specified by a fuzzy single ton, Therefore first order Sugeno fuzzy model is derived in this study to modeling the ANFIS algorithm for a first order rules Sugeno fuzzy inference system, the rules may be stated as:

$$
\begin{aligned}
& R_{1}: \text { If } x \text { is } A_{1} \text { and } y \text { is } B_{i} \text { then } f_{1 i}=r_{1 i} \\
& R_{2} \text { : If } x \text { is } A_{2} \text { and } y \text { is } B_{i} \text { then } f_{2 i}=r_{2 i}
\end{aligned}
$$

where $x$ is the input (antecedent) variable and $A_{i}$ is the antecedent linguistic constants (the qualitatively defined functions).

Similarly, $y$ is the input (antecedent) linguistic variable and $\mathrm{Bi}$ is the consequent linguistic constants. The values of $x$ and $y$, and $A_{i}$ and $B_{i}$ are fuzzy sets defined in the domains of their respective base variables. The linguistic terms $A_{i}$ and $B_{i}$ are usually selected from sets of predefined terms, such as high depth, medium and low.

The rule base $R=\{R i / i=1,2 \ldots, K\}$ and the sets $A$ and $B$ constitute the knowledge base of the linguistic model. Each rule is regarded as a fuzzy relation (fuzzy restriction on the simultaneous occurrences of values $x$ and $y$ ) $R i(x, y)$ Fuzzy number describes the relationship between an uncertain quantity $x$ and a membership function $(\lambda)$ which ranges between 0 and 1 . All the input membership 
functions $\left(\lambda_{A i}(x)\right)$ are Gaussian Membership Functions given by:

$$
\lambda_{A i}(x)=\frac{1}{\left(1+([x-c i / a i])^{2 b i}\right)}
$$

or

$$
\lambda_{A i}(x)=\exp \left(-\left(\frac{x-c i}{a i}\right)^{2}\right)
$$

where, $a_{i}$ and $b i$ are the lower and upper limits of the integral which determines the validity domain of the membership function. This relation can be computed in two basic ways by using fuzzy implications or fuzzy conjunctions. In this study, the Sugeno method was used, in which conjunction $A \wedge B$ is computed by a minimum operator.

$$
\begin{aligned}
\text { i.e. } R i=\left(A_{i} . B_{i}\right), \\
\qquad \lambda_{R i}(x, y)=\left[\lambda_{A i}(x) \Lambda \lambda_{B i}(y)\right]
\end{aligned}
$$

The minimum operator is computed on the Cartesian product space of $x$ and $y$, i.e., for all possible pairs of $x$ and $y$. The fuzzy relation $R$ represents the entire model (Equation (3)) and is given by the disjunction (union or maximum, i.e., s-norms) of the (n) individual rule relations $R_{i}$ :

$$
\begin{aligned}
& n=\bigcup_{i=1}^{n} R_{i} \\
& \lambda_{R}(x, y)=\max _{1 \leq i \leq n}\left[\lambda_{A i}(x) \Lambda \lambda_{B i}(y)\right]
\end{aligned}
$$

Now the entire base is encoded in the fuzzy relation $R$ and the output of the linguistic model can be computed by the max-min composition $\left(^{\circ}\right)$ :

$$
y=x^{o} R
$$

Suppose an input fuzzy value $x=A$ which has the output value $B^{\prime}$ given by the relational composition:

$$
\lambda_{B}(x, y)=\max _{x}\left[\lambda_{A}(x) \Lambda \lambda_{R}(x, y)\right]
$$

By substituting Equation (3) into Equation (6), get:

$\lambda_{B^{\prime}}(x, y)=\max _{x}\left[\lambda_{A^{\prime}}(x) \Lambda \max _{1 \leq i \leq n}\left[\lambda_{A i}(x) \Lambda \lambda_{B i}(y)\right]\right]$

Assuming $B i=\max _{x}\left[\lambda_{A}(x) \Lambda \lambda_{A i}(x, y)\right]$ is the degree of fulfillment of the (ith) rule's antecedent. The output fuzzy set of the linguistic. The output fuzzy set of the linguistic model is thus

$$
\lambda_{B^{\prime}}(y)=\max _{x}\left[B i \Lambda \lambda_{B i}(y)\right]
$$

Equation (8) represents the output fuzzy Set of the linguistic model.

The most common methods used for defuzzification is Yager's censorial method, is always used for defuzzifica- tion due to its simplicity that can be determined as:

$$
\text { Defuzzified value }(u)=\frac{\int_{a}^{b} y \cdot \lambda_{B^{\prime}}(y)}{\int_{a}^{b} \lambda_{B^{\prime}}(y)}
$$

where: $y$ is the censorial distance from the origin.

This algorithm is for single input and single output (SISO). It can be extended to multiple inputs and single output (MISO) and multiple outputs (MIMO). The MIMO model is a set of MISO models.

In this study, the predicting of removal efficiency should have been multi input and one output, therefore (MISO) model was used as follow:

Ri: If $x_{1}$ is $A_{1 i}$ and $x_{2}$ is $A_{2 i}$ and $x_{p}$ is $A_{p i}$ then $f$ is $r_{i}$

The above model is the special case of (Equation (1)), as the set $A_{i}$ is obtained by the Cartesian product of fuzzy sets:

$$
\begin{gathered}
A_{i}=A_{i 1} \times A_{i 2} \times A_{i 3} \times \ldots \ldots \ldots . . . \times A_{i p} \\
j=1,2 \ldots . p
\end{gathered}
$$

Hence the degree of fulfillment $\left(B_{i}\right)$ becomes:

$$
B i=\lambda_{\text {Ai1 }}\left(x_{1}\right) \Lambda \lambda_{\text {Ai2 }}\left(x_{2}\right) \Lambda \ldots \ldots . \Lambda \lambda_{\text {Aip }}\left(x_{p}\right)
$$

\section{Removal Efficiency Modeling}

\subsection{Data Set for Training and Validating}

The data collected for 575 operating conditions were used for developing a fuzzy rule-based model to predict the optimum removal efficiency of multimedia filter .The control parameters of (FLC) with Sugeno model, are seven parameters which are media depths, media grain size, filtration rate, particle diameter, feed concentration, and operation time. The output parameter is removal efficiency of media filter whereas these data are collocated from my experimental work of deep bed filter and for multimedia filter the overall removal efficiency was determined.

From the derivation as follow:

$$
\begin{gathered}
E_{i-1}=\frac{C_{0}-C_{G A C}}{C_{0}} \\
E_{i}=\frac{C_{G A C}-C_{i+1}}{C_{G A C}}
\end{gathered}
$$

From Equation (13)

$$
C_{G A C}=C_{0}\left(1-E_{i-1}\right)
$$

Substituted Equation (15) into Equation (14) yields:

$$
E_{i}=\frac{C_{0}\left(1-E_{i-1}\right)-C_{i+1}}{C_{0}\left(1-E_{i-1}\right)}
$$


The overall efficiency ( $E_{\text {filter }}$ of filter is equal to sum of efficiency for all layer in depth filter, thus can be express as:

$$
E_{\text {filter }}=E_{i-1}+E_{i}\left(1-E_{i-1}\right)
$$

where, $C_{i+1, i+2, \ldots . . . N}$ indicate to sand bed, $i=$ The number of output layer.

The mean and standard deviation of the inputs and output were used to define the granularities (the levels of scale). The variability in the input control parameter under any given condition was used for fuzzificaion. A two granule scale, low and high, was used for operation time.

Similarly, feed concentration of dispersed particles and filtration rate were expressed by three (low, medium, high) and four granule scale for media depths (very low, low, medium, high), finally five scale was taken for diameter of particle (very low, Medium, high, vary high) as shown in Figures 3(a)-(g).

The fuzzy rule based model was developed in the following format:

R1: If $x$ is high and $y$ is Low and ......then $E$ is ......

$R 2$ : If $x$ is Low and $y$ is high and ...then $E$ is ......

\subsection{Modeling Results}

In this study, the input parameters are subdivided into several types of reference fuzzy sets with the 575 training dataset, we choose some of these rules and inputs parameter to build the last structure of ANFIS model as shown Figure 4 after the model was trained, the inference was performed in accordance with 575 rule of FLC.

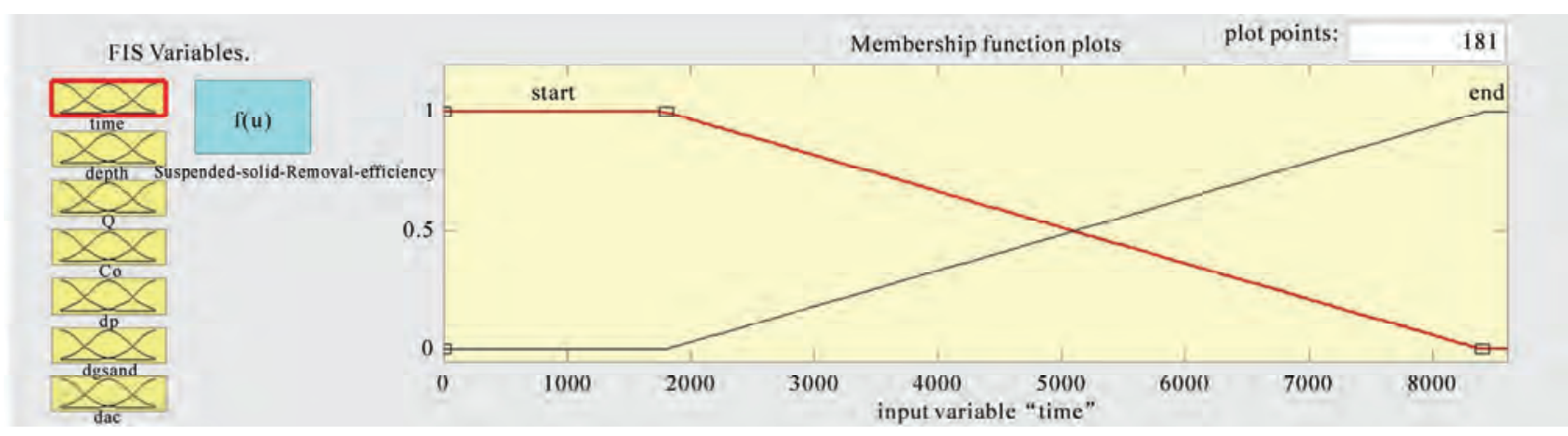

(a)

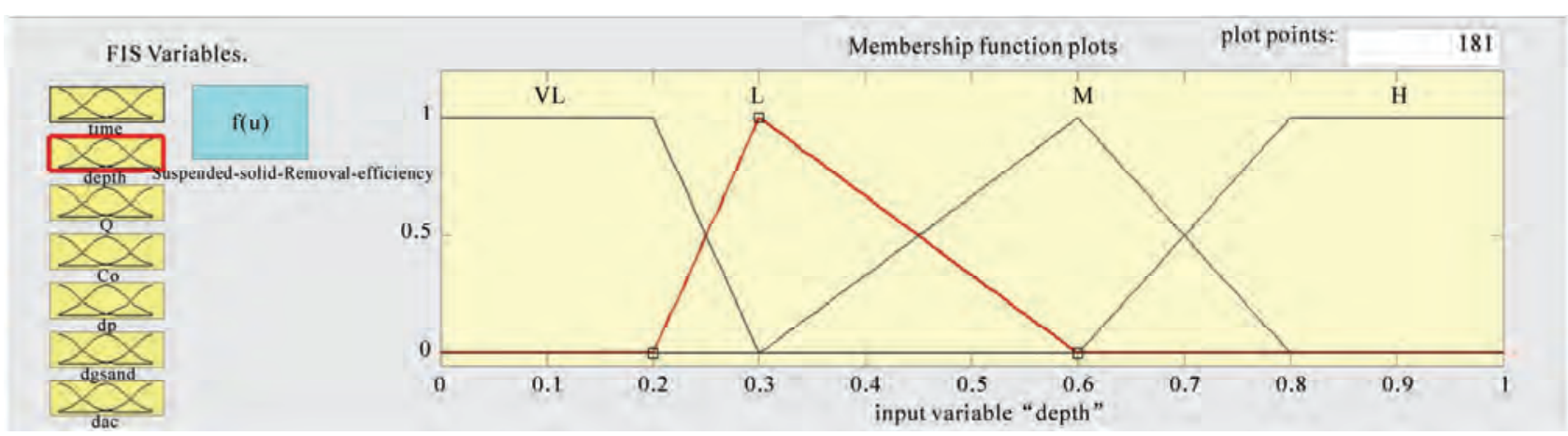

(b)

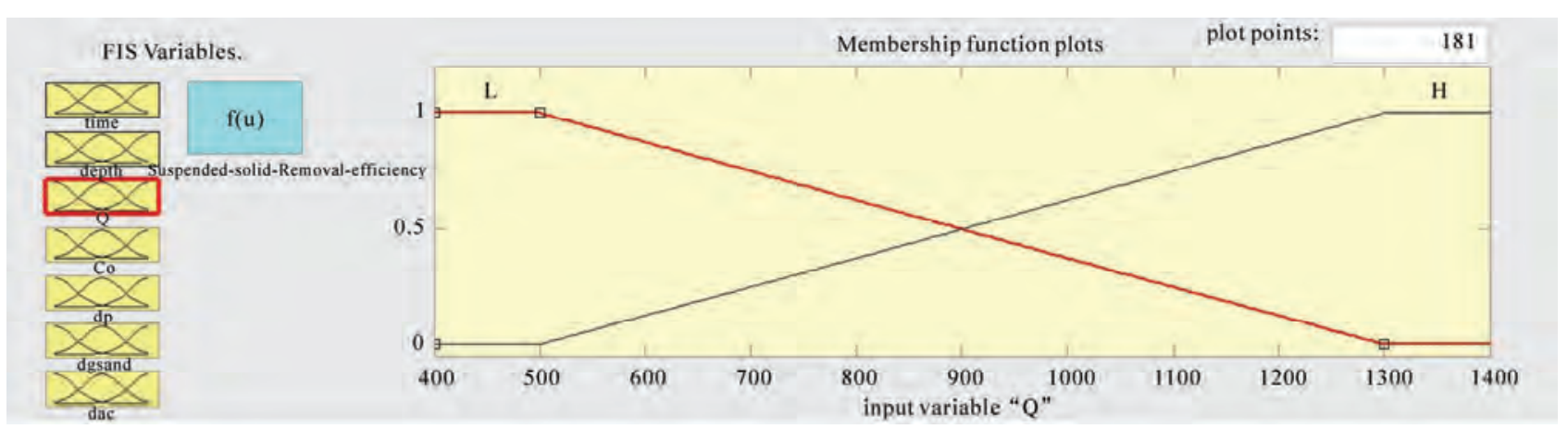

(c) 


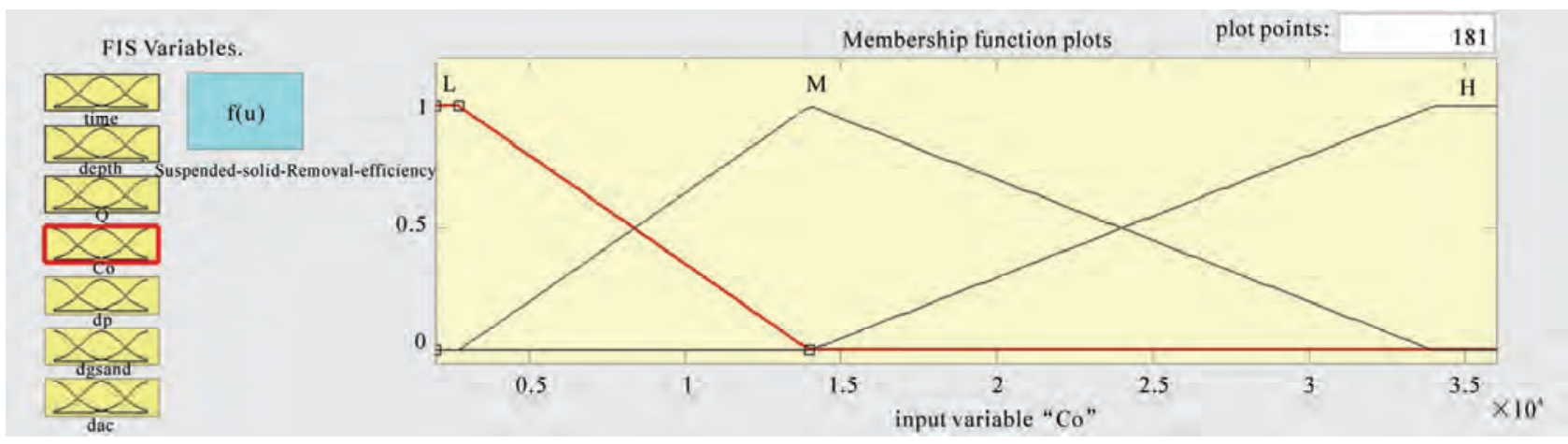

(d)

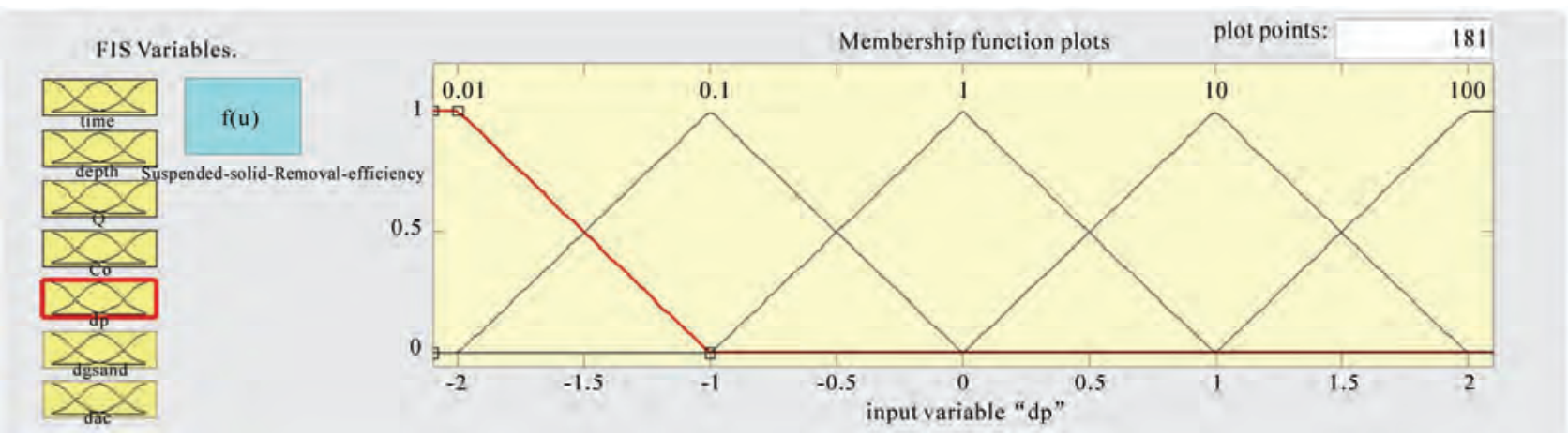

(e)

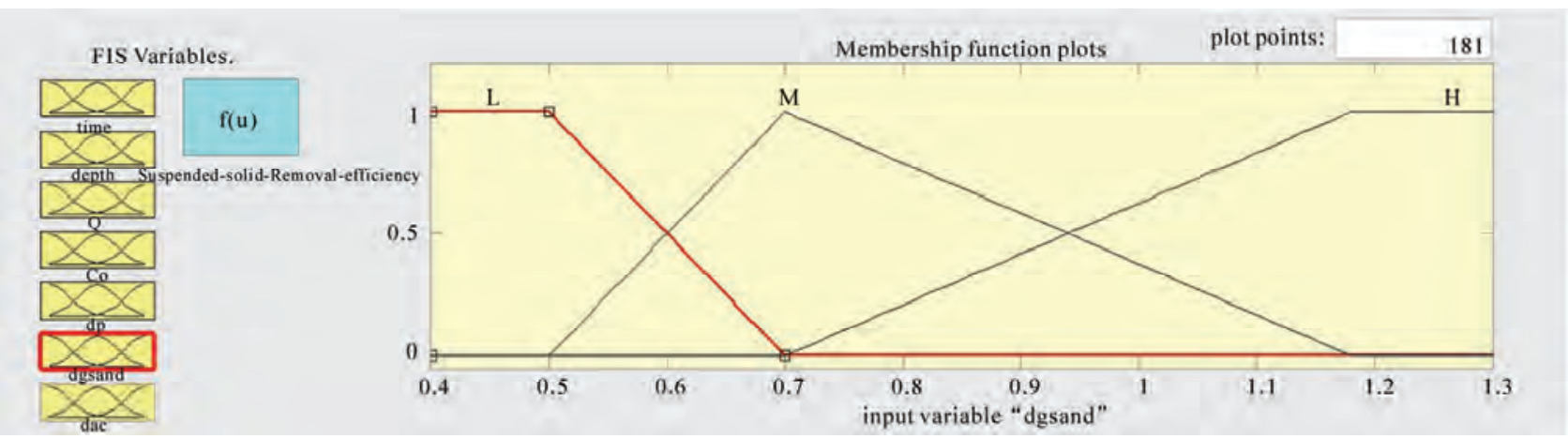

(f)

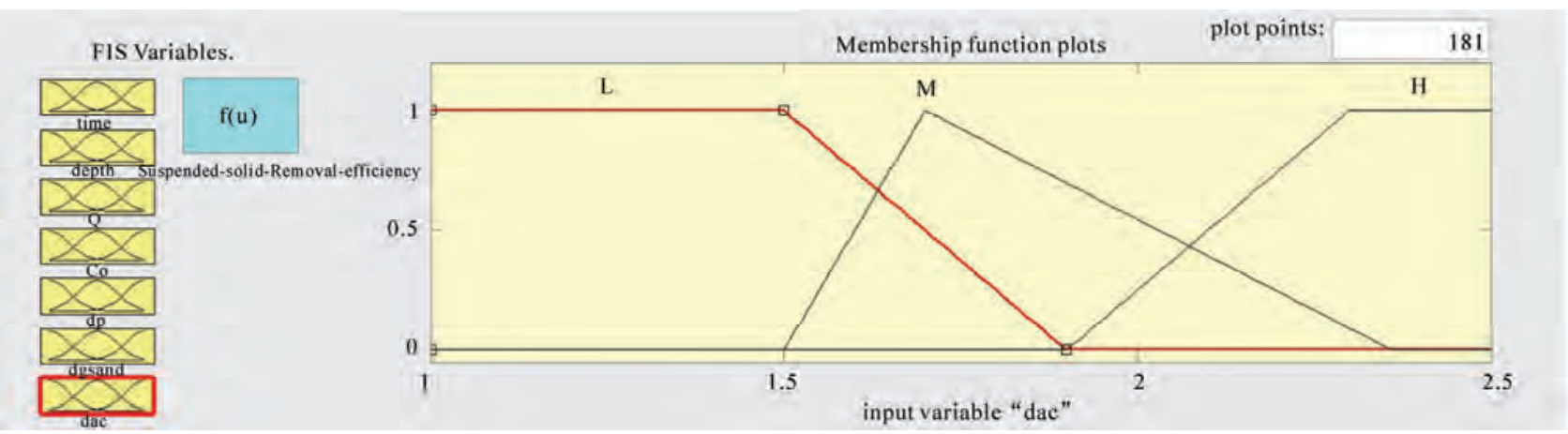

(g)

Figure 3. (a)-(g) The qualitative scales for inputs and output of (MISO) model. 


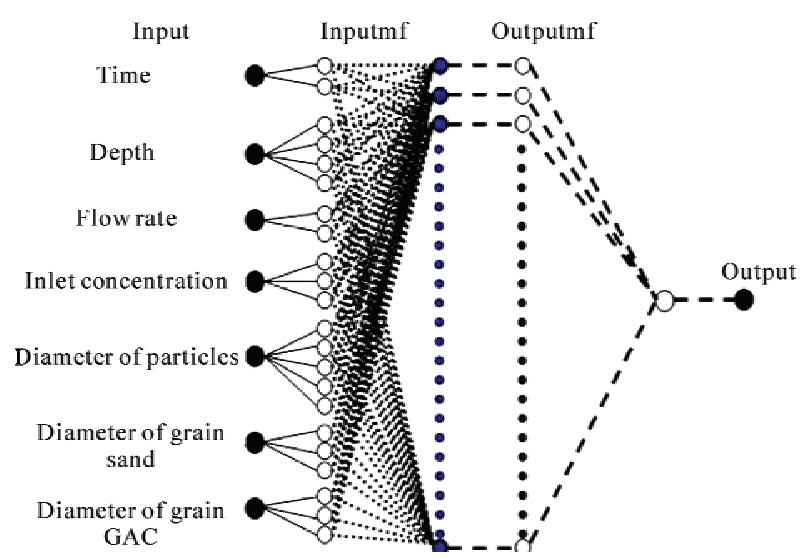

Figure 4. ANFIS structure for removal efficiency.

These rules are applied with deferent boundary condition to predict the removal efficiency (Figure 5). Those rules were obtained after the network was trained. Some other rules were also included theoretically in terms of comparing output values in accordance with input values.

Figures 6(a)-(b) shows the results of applied rules and their corresponding outputs according to the input date that should been determined. Using the interface, defuzzified values for output variables can be derived by changing input values manually. We selected the input data flow rate, $500-1300 \mathrm{~cm}^{3} / \mathrm{min}$, bed height $0.5-0.8 \mathrm{~m}$, feed concentration of dispersed particles is $14000-34000$ $\mathrm{mg} / \mathrm{l}$, grain size is $0.5-0.7 \mathrm{~mm}$ of sand and $1.5-1.9 \mathrm{~mm}$ of GAC with 600-8400 second of operation time, finally diameter of particle is $0.01-10 \mu \mathrm{m}$. the removal efficiency of multimedia filter is predicted by FLC that equal to $96.9 \%$ and $80 \%$ as shown in Figures 6(a)-(b) respectively. It is not flexible to get defuzzified output values for all the real input values using the interface. For that reason, a program is written using Matlab codes to drive defuzzified output results in accordance with real input values.

Figures 7(a)-(g) shows the result of prediction the removal efficiency of multimedia filter and the inputs variable by Surgeon system. These results are plotted by three dimensional graphic which illustrates several surface viewer that obtained from the fuzzy logic toolbox.

\subsection{Simulation Result by (ANFIS) Model}

In this study, the final adaptive of neuron inference system (ANFIS) was proposed by of training data set that show perversely. (ANFIS) model was employed to predict the removal efficiency of suspended solids with respect to sample number. The performance of this prediction was evaluated based on mean absolute percentage error (MAPE) as follow:

$$
M A P E=\frac{1}{N} \sum_{t=1}^{N}\left|\frac{y_{\exp }-y_{p}}{y_{p}}\right| \times 100
$$

Another evaluation is used in (ANFIS) model is the correlation coefficient $(\mathrm{R})$ which can be defined as follow:

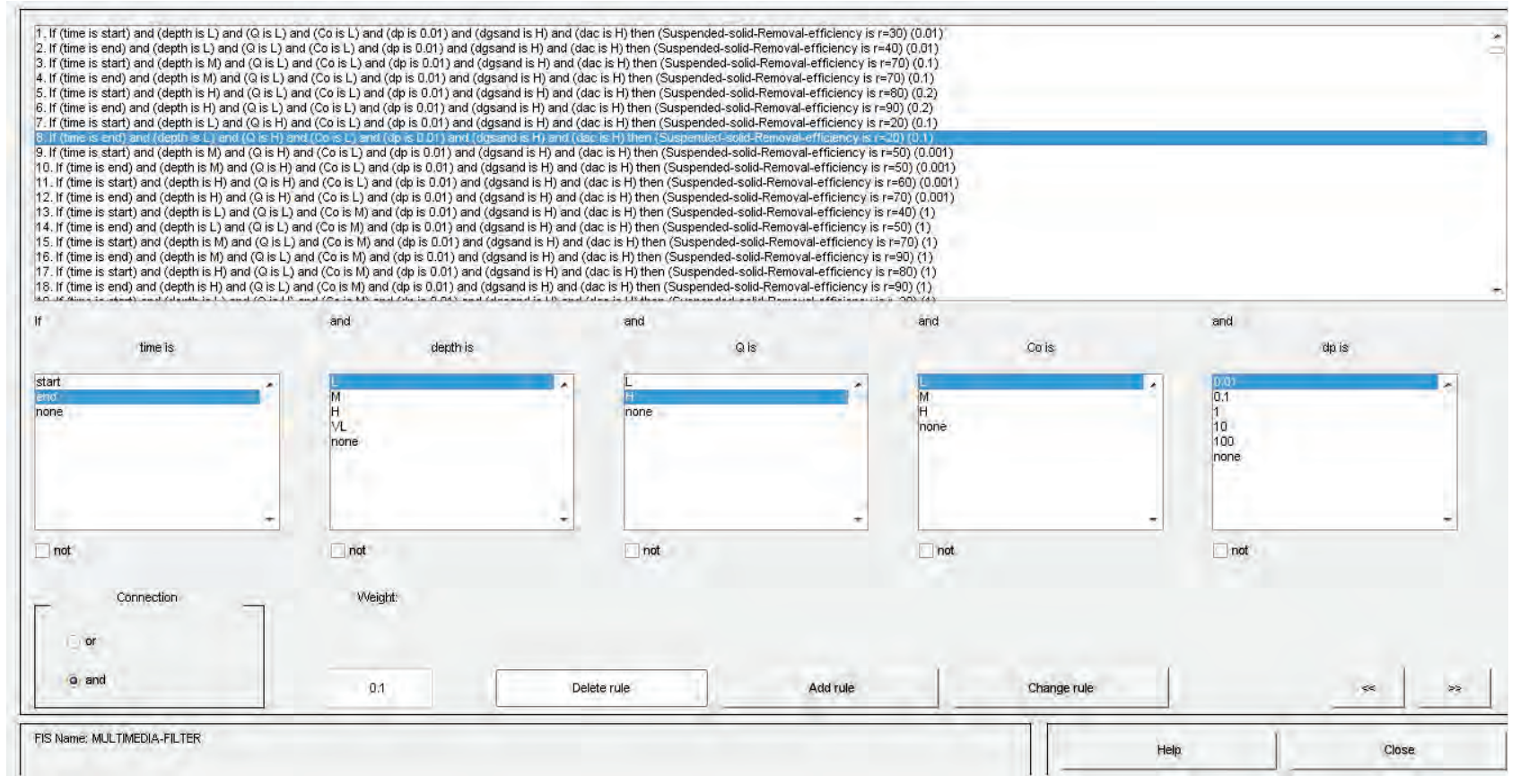

Figure 5. Rule editor of fuzzy logic control tool. 


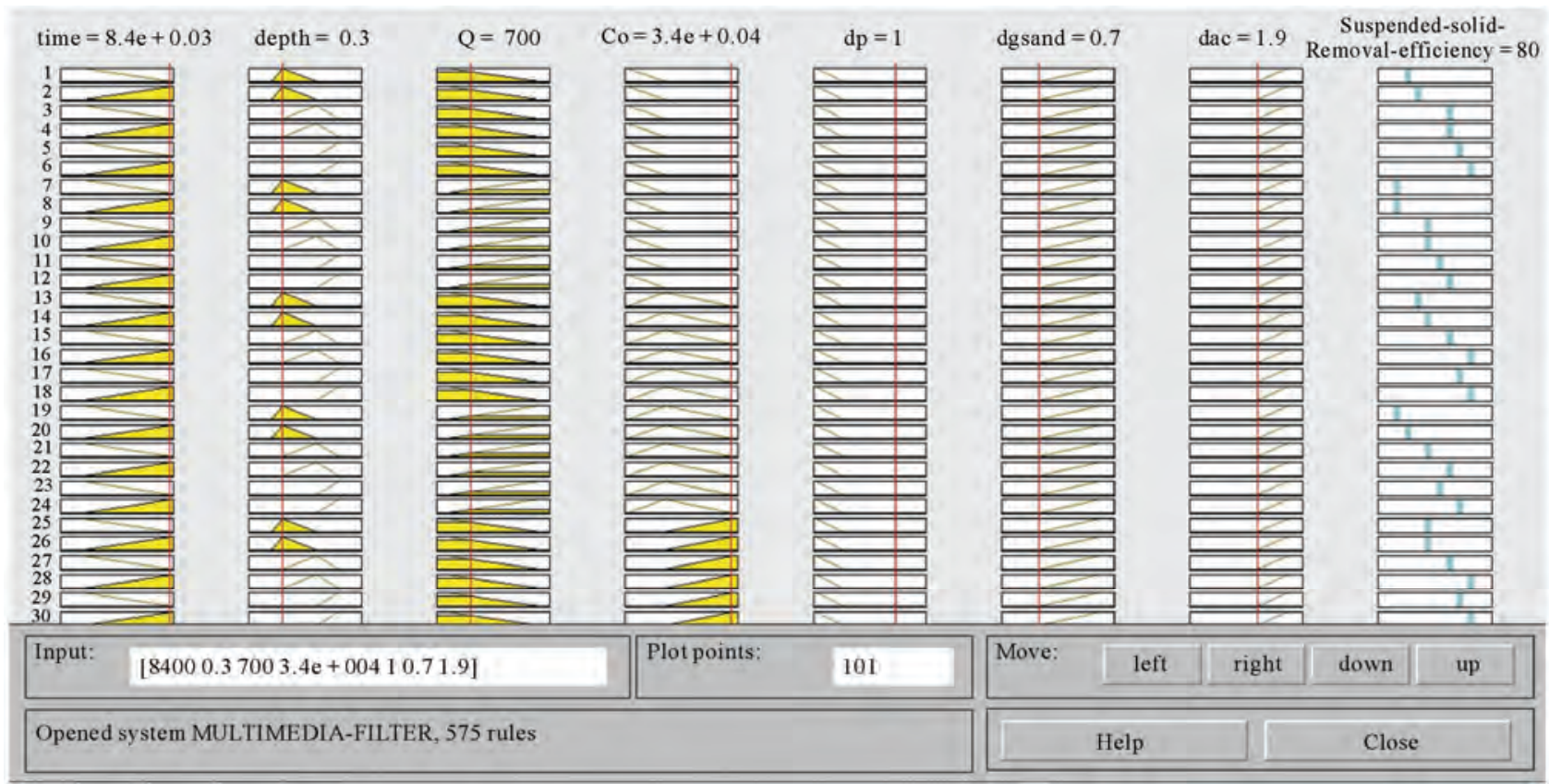

(a)

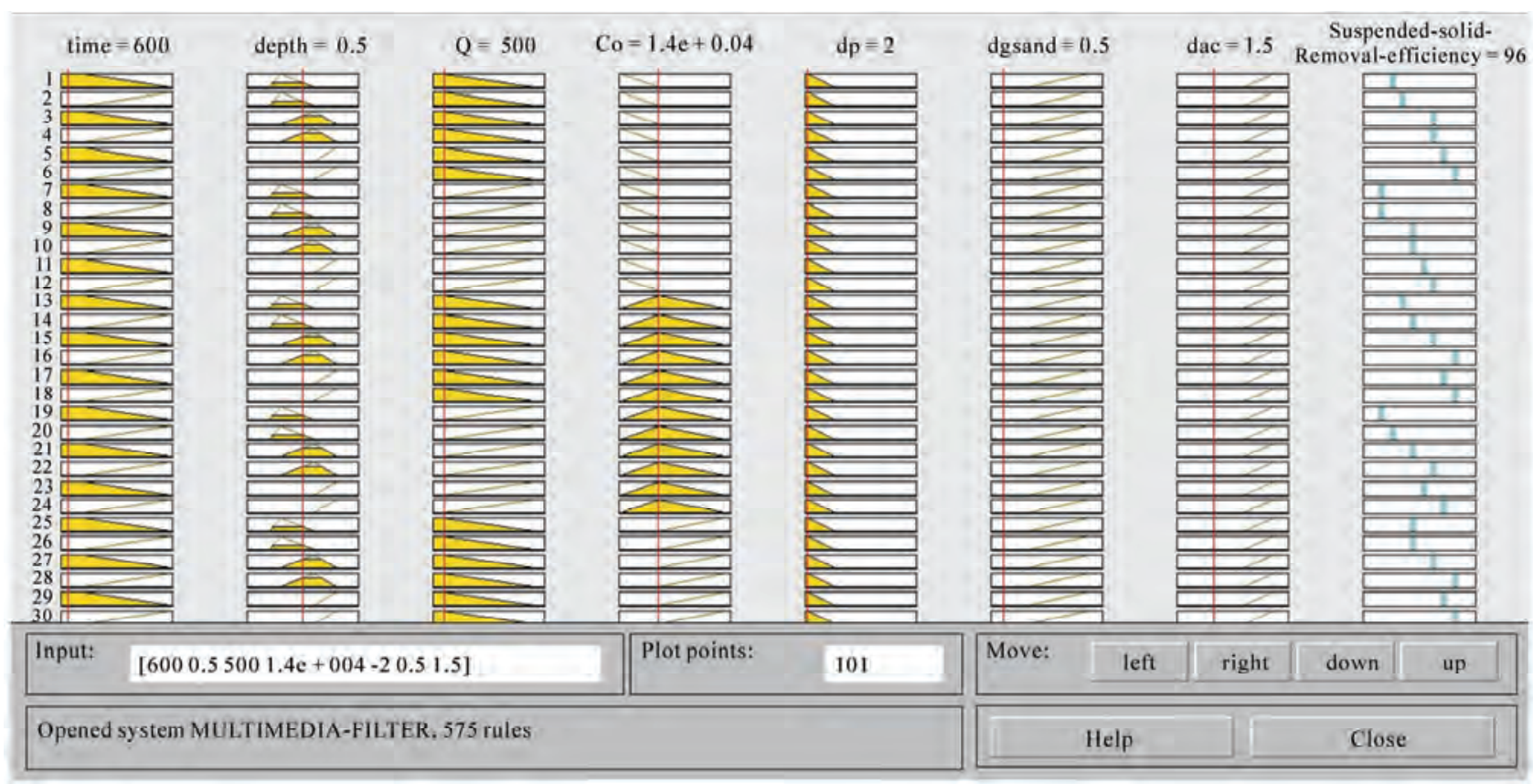

(b)

Figure 6. (a)-(b) Rule viewer screen to obtain of defuzzified results.

$$
R=\frac{\sum_{t=1}^{N}\left(y_{\exp }-\bar{y}\right)\left(y_{p}-\bar{y}_{p}\right)}{\sqrt{\sum_{t=1}^{N}\left(y_{\exp }-\bar{y}\right)^{2} \sum_{t=1}^{N}\left(y_{p}-\bar{y}_{p}\right)^{2}}}
$$

Figure 8 shows the simulation result, between the experimental data and the prediction of (ANFIS) model. The best performance is achieved by MAPE that equal
7.0458 .

\section{Conclusions}

Modeling of filter performance is very difficult because of complexity of the defining physical and chemical events in the filtration system whereas the knowledge of functionality of filter coefficient. So, in this study, AN 


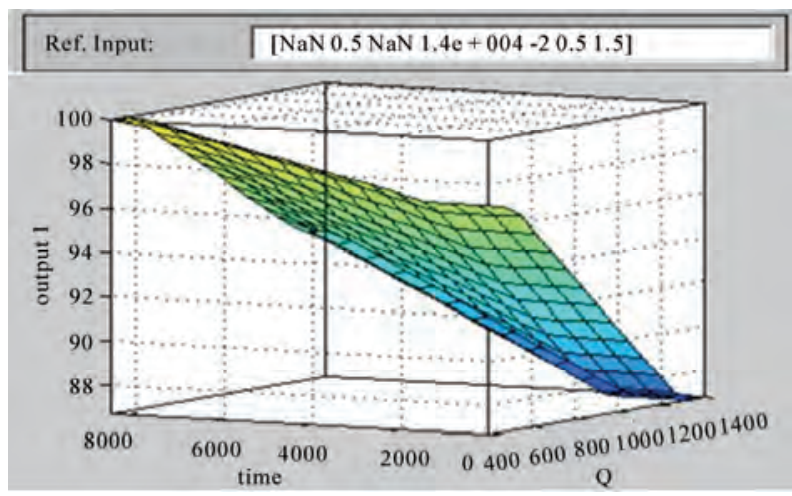

(a)

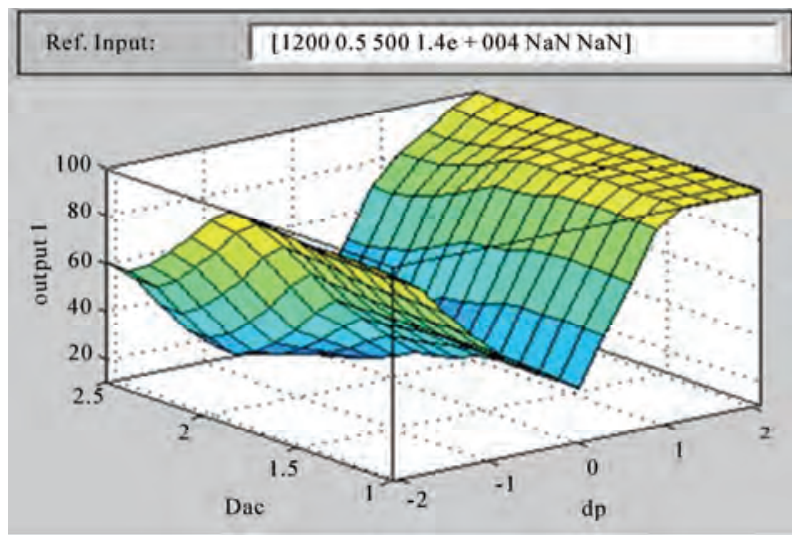

(b)

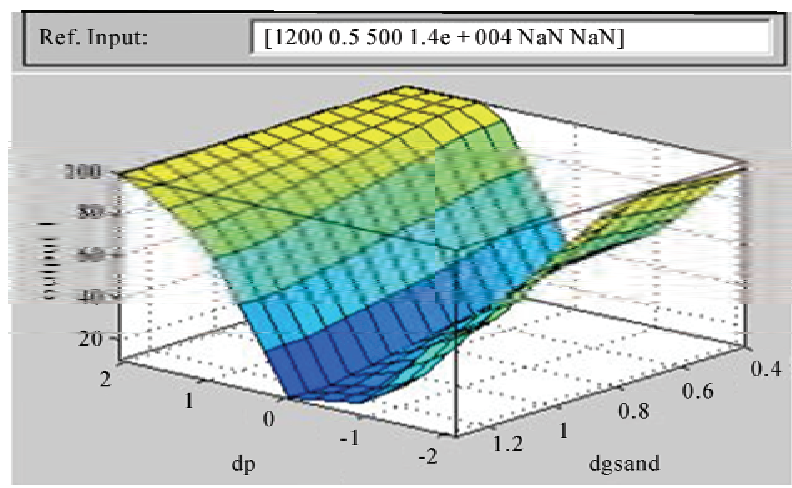

(c)

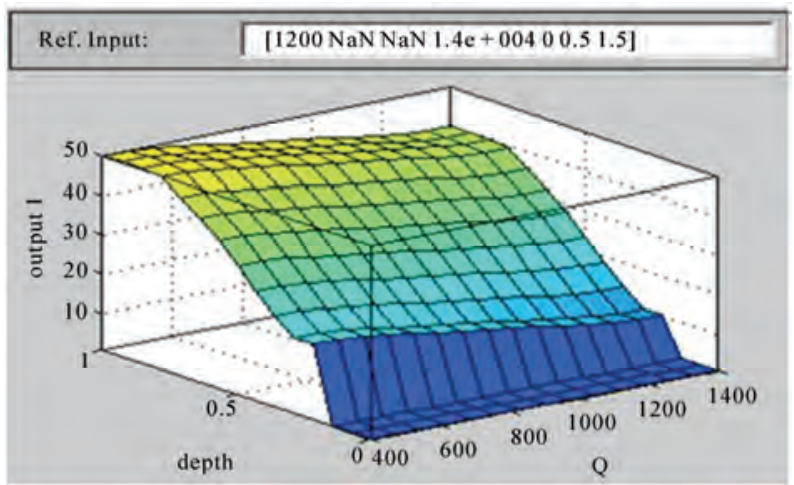

(d)

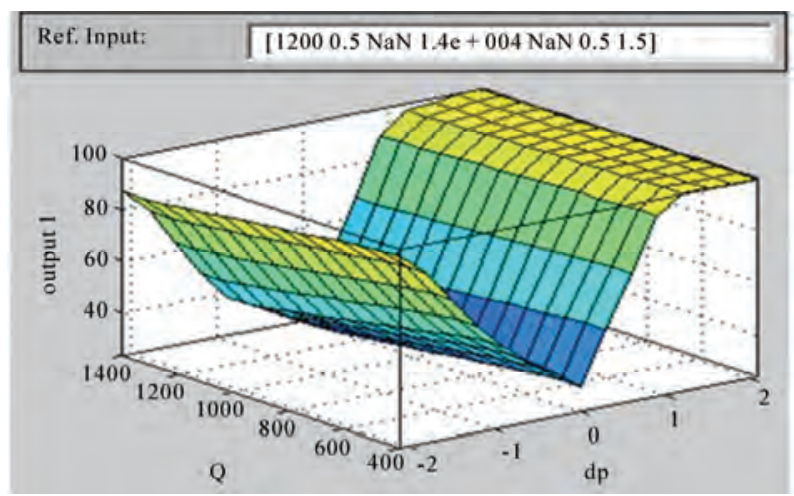

(e)

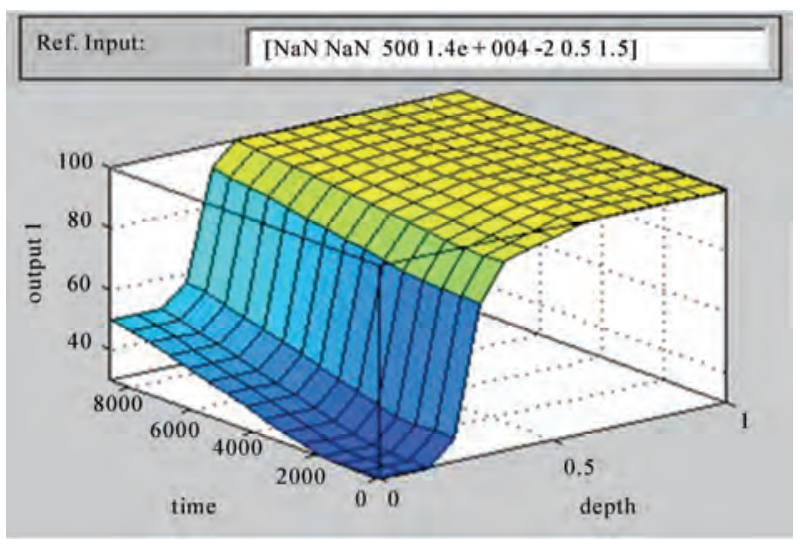

(f)

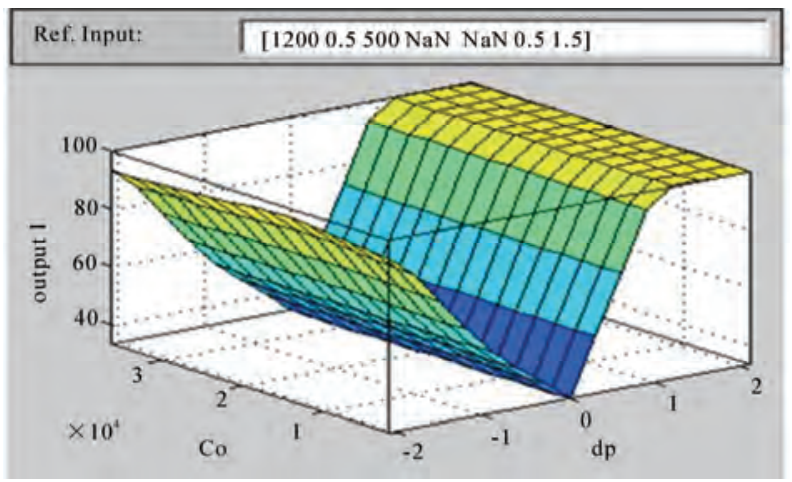

(g)

Figure 7. (a)-(g) 3-D Response surface graph for removal efficiency with different input parameter.

FIS models were used to predict the performance of multimedia filter. The results of this study can be depicted as follows:

1) The fuzzy logic control model (FLC) was established in multimedia deep bed filter to predict the performance of multimedia filter (sand + GAC) and to evaluate both initial and transient stage of suspension particle removal. Whereas the result showed that it is a flexible tool to study the performance of multimedia filter for dispersed particles removal in terms of different operating 


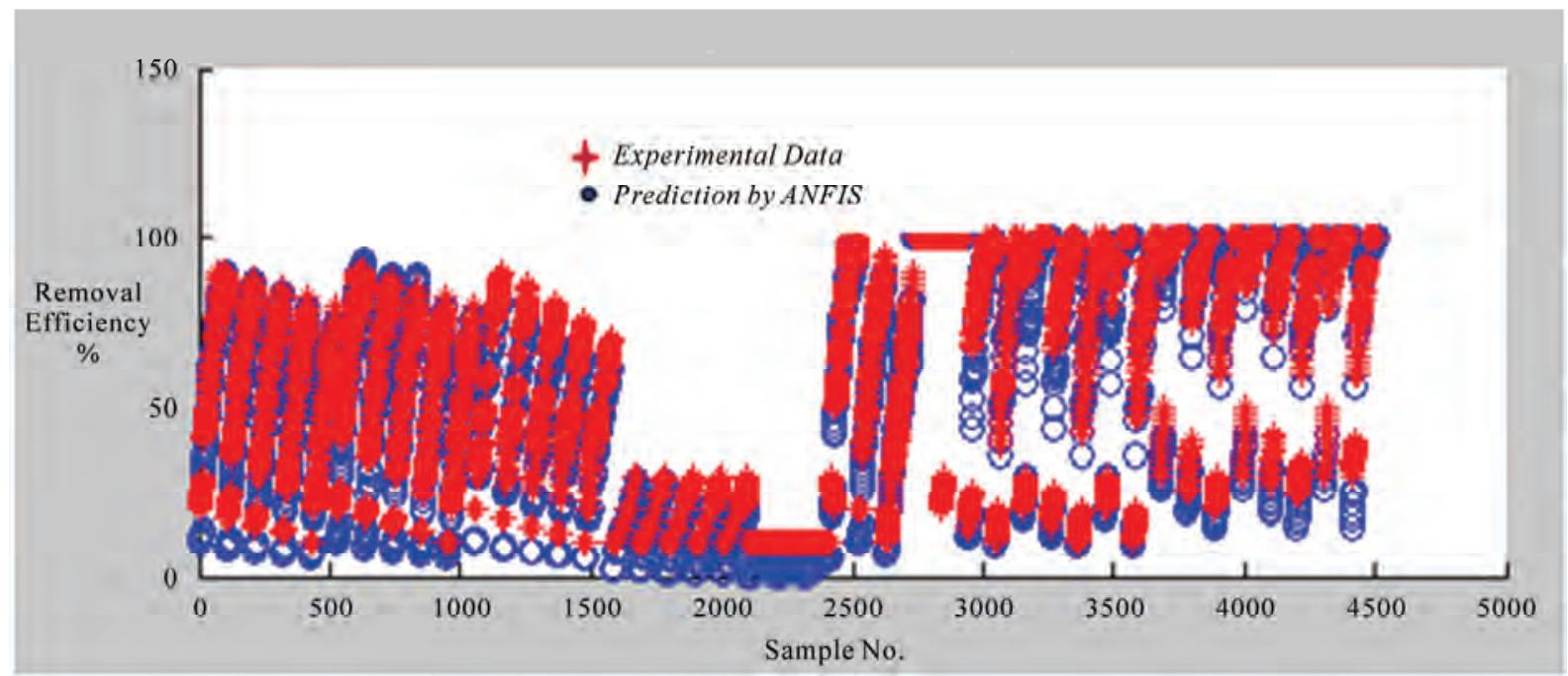

Figure 8. Simulation result of removal efficiency by (ANFS) model.

parameter whereas the optimal removal efficiency 95100 achieved by $0.5-0.7 \mathrm{~mm}$ grain size of sand, 1.5-1.9 mm grain size of GAC, with media depths should range from 0.3 to $0.6 \mathrm{~m}$.

2) The overall removal efficiency depends on filtration velocity, physical properties of media, media type and the quality of row water, the result illustrates that there is a relationship between flow rate, effective size and optimum bed depth. For all filter media, the increment of effective size generates a higher value of optimum filter bed depth for a lower value of filtration rate; in addition to that the high removal efficiency can be achieved by using smaller grain size.

3) Adaptive Nauruan fuzzy inference system (ANFIS) was used to simulate the experimental result. The Simulation result showed a good agreement between observed and predicted values for both training and testing data whereas the MABE is equal to 7.0458.

\section{REFERENCES}

[1] C. T. Alan, D. R. Don and J. B. Malcolm, "Water Supply,” 5th Edition, 2000, pp. 335-347.

[2] K. Yao, M. T. Habiban and C. R. O’Melia, "Water and Wastewater Concept and Applications," Environmental Science Technology, Vol. 5, No. 11, 1971, pp. 1105-1112.

[3] C. Tien and B. V. Remoarao, "Granuler Filtration of Aersols and Hydrosols,” 2007, pp. 29-31.

[4] A. Salim, S. Sizert and A. Bruand. "Development of Adarcy Brinkman Model to Simulate under Flow and Tracer Transport in a Heterogeneous Karstic Aquifer," Hydrogeology Journal, Vol. 15, 2008.

[5] Y. Sung and Andrew "Simulation of Pore-Scale Particle Deposition and Clogging," Journal of Transpiration in Porous Media, Vol. 65, No. 11, 2005, pp. 53-87.
[6] L. A. Zadeh, "Fuzzy Logic Computing with Words," IEEE Transactions-Fuzzy Systems, Vol. 4, No. 2, 1996, pp. 103-111.

[7] S. R. Al-Zahrani, M. A. Sheikh, A. K. T. Husain and S. Farooq, "Performance Evaluation of Slow Sand Filters Using Fuzzy Rule-Based Modeling," Environmental Modeling \& Software, Vol. 19, No. 5, 2003, pp. 507-515.

[8] A. Altunkaynak, M. Ozger and M. Cakmakci, "Fuzzy Logic Modeling of the Dissolved Oxygen Fluctuations in Golden Horn,” Ecological Modelling, Vol. 189, No. 34, 2005, pp. 436-446.

[9] O. Terzi, M. E. Keskin and E. D. Taylan, "Estimating Evaporation Using ANFIS," Journal of Irrigation and Drainage Engineering - ASCE, Vol. 32, No. 5, 2006, pp. 503-507.

[10] A. Altunkaynak, M. Özger and M. Cakmakci, “Consümption Prediction of Istanbul City by Using Fuzzy Logic Approach," Water Resources Management, Vol. 19, No. 5, 2005b, pp. 641-654.

[11] F. J. Chang and Y. T. Chang, “Adaptive Neuro-Fuzzy Inference System for Prediction of Water Level in Reservoir," Advances in Water Resources, Vol. 29, No. 1, 2005(b), pp. 1-10.

[12] M. Cakmakci, "Adaptive Neuro-Fuzzy Modeling of Anaerobic Digestion of Primary Sedimentation Sludge," Bioprocess and Biosystems Engineering, Vol. 30, No. 5, 2005(b), pp. 349-357.

[13] S. R. Jang, “ANFIS: Adaptive-Network-based Fuzzy Inference Systems," IEEE Transaction on Systems, Man and Cybernetics, Vol. 23, No. 3, 1993, pp. 665-685.

[14] M. Z. Huang, J. Q. Wan, Y. W. Ma, and W. J. Li, “A Fast Predicting Neural Fuzzy Model for On-Line Estimation of Nutrient Dynamics in an Anoxic Process," Journal of Science Direct Bioresource Technology, Vol. 101, No. 6, 2010, pp. 1642-1651. 